\title{
Magnetic White Dwarfs in the SDSS and Estimating the Mean Mass of Normal DA and DB WDs
}

\author{
S.O. Kepler*, S.J. Kleinman ${ }^{\dagger}$, Ingrid Pelisoli*, Viviane Peçanha*, Marcos \\ Diaz $^{* *}$, Detlev Koester ${ }^{\ddagger}$, B. G. Castanheira ${ }^{\S}$ and Atsuko Nitta ${ }^{\dagger}$ \\ ${ }^{*}$ Instituto de Física da UFRGS, Brazil \\ ${ }^{\dagger}$ Gemini Observatory, Hawaii, USA \\ ** Instituto Astronômico e Geofísico da USP, Brazil \\ ${ }^{\ddagger}$ Institut für Theoretische Physik und Astrophysik, Universität Kiel, Kiel, Germany \\ ${ }^{\S}$ Institut für Astronomie, Wien, Austria
}

\begin{abstract}
When classifying by eye more than 22000 spectra selected as possible white dwarf stars from the Sloan Digital Sky Survey Data Release 7, we detected Zeeman splittings in more than 800 stars, increasing by a factor of five the number of known magnetic white dwarfs. Our field estimations range from $90 \mathrm{MG}$ to less than $1 \mathrm{MG}$, complementing the detections by Külebi et al. [1]. These magnetic white dwarf stars cover the whole range of temperature and spectral classes observed.

As the Zeeman splittings broadens the lines, we cannot use the line profiles to estimate surface gravity directly. We therefore excluded the magnetic white dwarfs from our average mass estimate of normal DAs and DBs. Analysis of the remaining 1505 bright and hot DA white dwarfs, i.e., those with $S / N \geq 20$ and $T_{\text {eff }}=12000 \mathrm{~K}$, results in a mean mass $\langle M\rangle_{\mathrm{DA}}=0.604 \pm 0.003 M_{\odot}$, while that of our 82 bright DBs with $T_{\text {eff }}=16000 \mathrm{~K}$ is $\langle M\rangle_{\mathrm{DB}}=0.646 \pm 0.006 M_{\odot}$.
\end{abstract}

Keywords: white dwarf stars; magnetic fields; masses

PACS: 97.10.Ex, 97.10.Ld, 97.10.Nf, 97.20.Rp

\section{MAGNETIC WHITE DWARF STARS}

Kleinman et al. [2] is forming a catalog of white dwarf stars with spectra from the Sloan Digital Sky Survey Data Release 7. Using careful human inspections of our more than 23,000 white dwarf spectra, much more than were looked at in past SDSS white dwarf catalogs, we found evidence of magnetic field - Zeeman splittings - in somewhere between $5-10 \%$ of our white dwarf sample. The vast majority of these stars are low field.

Independent of Kleinman et al. [2], Külebi et al. [1] found 44 new magnetic white dwarfs in the SDSS DR7 sample, and used $\log g=8.0$ models to estimate the fields of the 141 known magnetic white dwarfs (1 to $900 \mathrm{MG}$ ).

Donati \& Landstreet [3] discuss extensively that magnetic fields are found in all types of stars and play a role in their formation and evolution, influencing accretion, diffusion, mass loss, turbulence, pulsations, their rotation rates and even their masses and surface chemical composition. The fraction of white dwarf stars that are magnetic is quoted in the literature as anywhere between $2 \%$ to $20 \%$. An accurate estimate of this percentage is crucial to understanding the origin of the magnetic field in these stars. We find $5 \%$ of the SDSS white dwarf stars show clear evidence of magnetic fields with another $4 \%$

\footnotetext{
CP1273, $17^{\text {th }}$ European White Dwarf Workshop edited by M. K. Werner and T. Rauch

(C) 2010 American Institute of Physics 978-0-7354-0823-4/10/\$30.00
} 

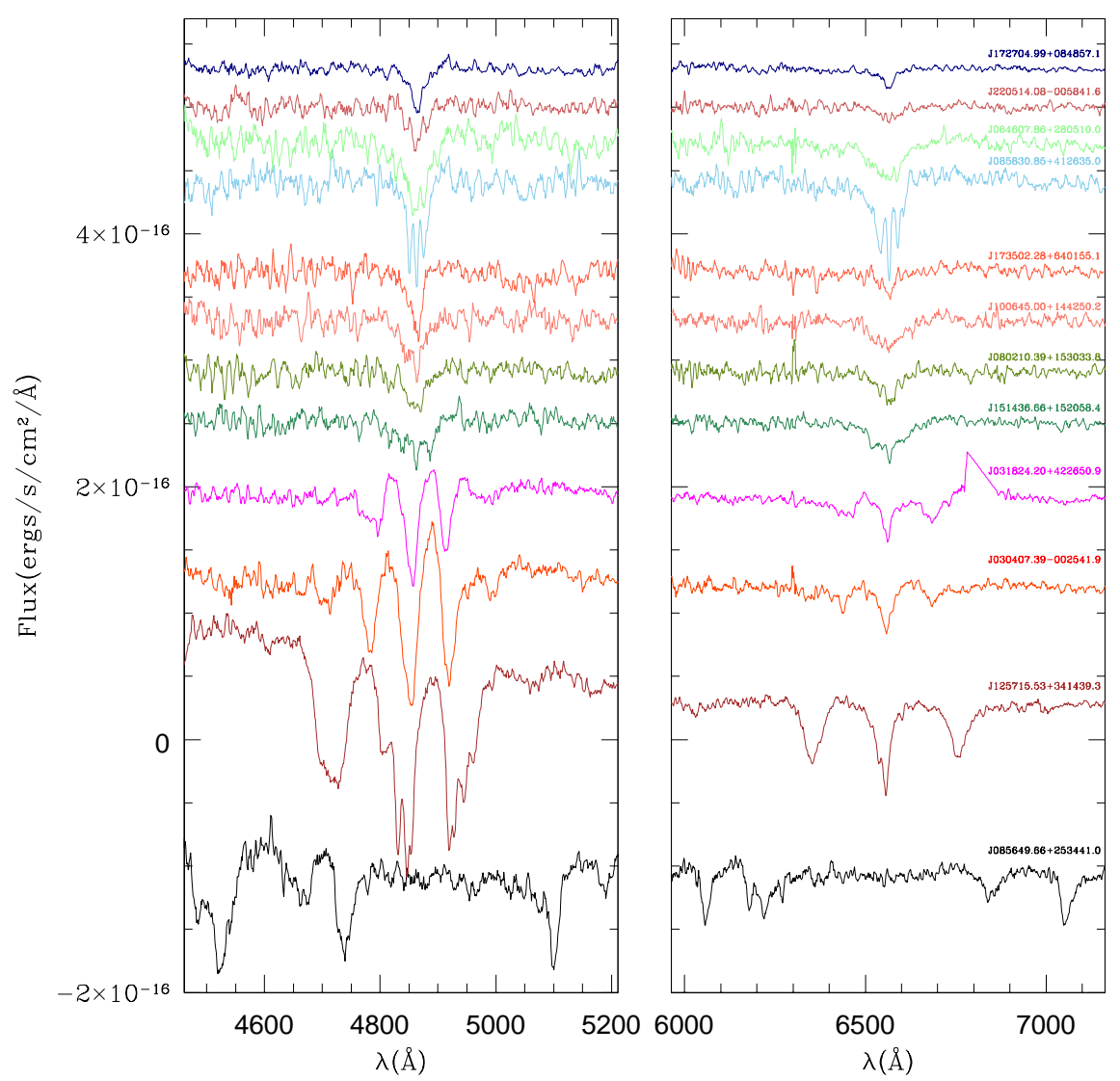

FIGURE 1. New magnetic white dwarf stars, with fields of $\simeq 2 \mathrm{MG}$ at the top, and $90 \mathrm{MG}$ at the bottom.

appearing possibly magnetic. Confirming the reliability of our determinations, and the number of possible magnetic white dwarf stars that truly are magnetic is necessary to refine our ratio and use it as a constraint on the origin of magnetic fields in white dwarf stars.

Single white dwarf masses are typically determined through spectroscopy - measuring line widths due to Stark pressure broadening. The problem is that Zeeman splitting, due to the possible presence of a weak magnetic field, if not clearly resolved, can mimic Stark broadening and an average mass white dwarf star, with a small magnetic field can appear indistinguishable, spectroscopically, from a non-magnetic, massive white dwarf star. The precise physics of exactly how magnetic fields affect line widths is not well understood. Depending on its strength, a magnetic field can alter the opacity of the 
layers, the radiative transfer, and the structure of the atmosphere, in addition to its direct effect on the line profiles.

The most common types of white dwarf stars are the DAs (hydrogen dominated atmosphere) and DBs (helium dominated atmospheres). A well-documented, but poorlyunderstood observed effect is the apparent increase in mass for cooler white dwarfs happens around $12000 \mathrm{~K}$ for DAs and $16000 \mathrm{~K}$ for DBs ([4], [5], [6], [7]). Mass determinations from photometry, seismology and gravitational redshift [8] do not show this mass increase, so we suspect the increase is not real, and merely reflects some missing physics in our spectroscopic models. Recently, there have been extensive efforts to improve the physics of the spectroscopic models, including a better treatment of the line broadening ([9], [10]), but the apparent mass increase remains. Could line broadening due to otherwise undetected magnetic fields be the cause of this apparent increase?

When we study the distribution of magnetic fields with effective temperature, we find an increase in the mean field around the same temperature when these stars develop a surface convection zone, raising the possibility that the surface convection zone is amplifying an underlying magnetic field.

The SDSS white dwarf sample provides us with the first statistically significant distribution of magnetic field versus temperature. It is necessary to investigate if surface convection amplification of an underlying weak magnetic field is causing broadening of the spectral lines of white dwarf stars cooler than $13000 \mathrm{~K}$, leading to misinterpretation of these stars as more massive stars.

In SDSS DR7 [2] we found 937 white dwarfs showing strong evidence of Zeeman splittings caused by magnetic fields of the order of 1 to $90 \mathrm{MG}$, plus 767 possibly magnetic stars. This corresponds to 5 to $9 \%$ of magnetic white dwarfs observed. Liebert et al. [11] found only $2 \%$ of the 341 DAs and 15 DBs in the PG survey are magnetic, but estimated that up to $10 \%$ could be magnetic, if the magnetic white dwarfs are more massive than average white dwarfs, as indicated by Liebert et al. [12] and Sion et al. [13]. Kawka et al. [14] estimated up to $16 \%$ of all white dwarfs may be magnetic and proposes that Ap and Bp stars, which exhibit large scale non variable fields, can only account for $4.3 \%$ (and producing white dwarfs with fields above $100 \mathrm{MG}$ ). Ap/Bp stars constitute less than $10 \%$ of all intermediate mass main sequence stars (e.g. Power et al. [15]) and using a normal initial to final mass relation (IFMR) should produce white dwarf stars with a mean mass around $0.6 M_{\odot}$, i,e., normal white dwarf masses. Some early B and O stars also show detectable magnetic fields. Jordan et al. [16], based on spectropolarimetry using the $8.2 \mathrm{~m}$ telescope VLT at ESO estimate up to 15 to $20 \%$ of all white dwarfs are magnetic at the kG level. Braithwaite \& Spruit [17], Tout et al. [18] and Wickramasinghe \& Ferrario [20] argue the fields are fossil. The last paper quotes a mean mass of $0.93 M_{\odot}$ for magnetic white dwarfs, compared with $0.6 M_{\odot}$ for normal white dwarfs, based on Liebert et al. [11] determinations, but the sample includes only a handful of stars with directly measured masses, so the evidence that the magnetic white dwarfs are more massive than the average is based on an statically insignificant number of stars. Liebert et al. [4] found no magnetic white dwarf from the 106 known in the 2551 new white dwarfs in DR4 were in noninteracting system with main sequence stars, while $25 \%$ of the known cataclysmic variables are magnetic ([19]) and around $19 \pm 4 \%$ of the white dwarfs in the 20 pc sample are magnetic. Our sample includes 110 possible magnetic white dwarf+main sequence pairs, all with fields estimated below $3 \mathrm{MG}$. 


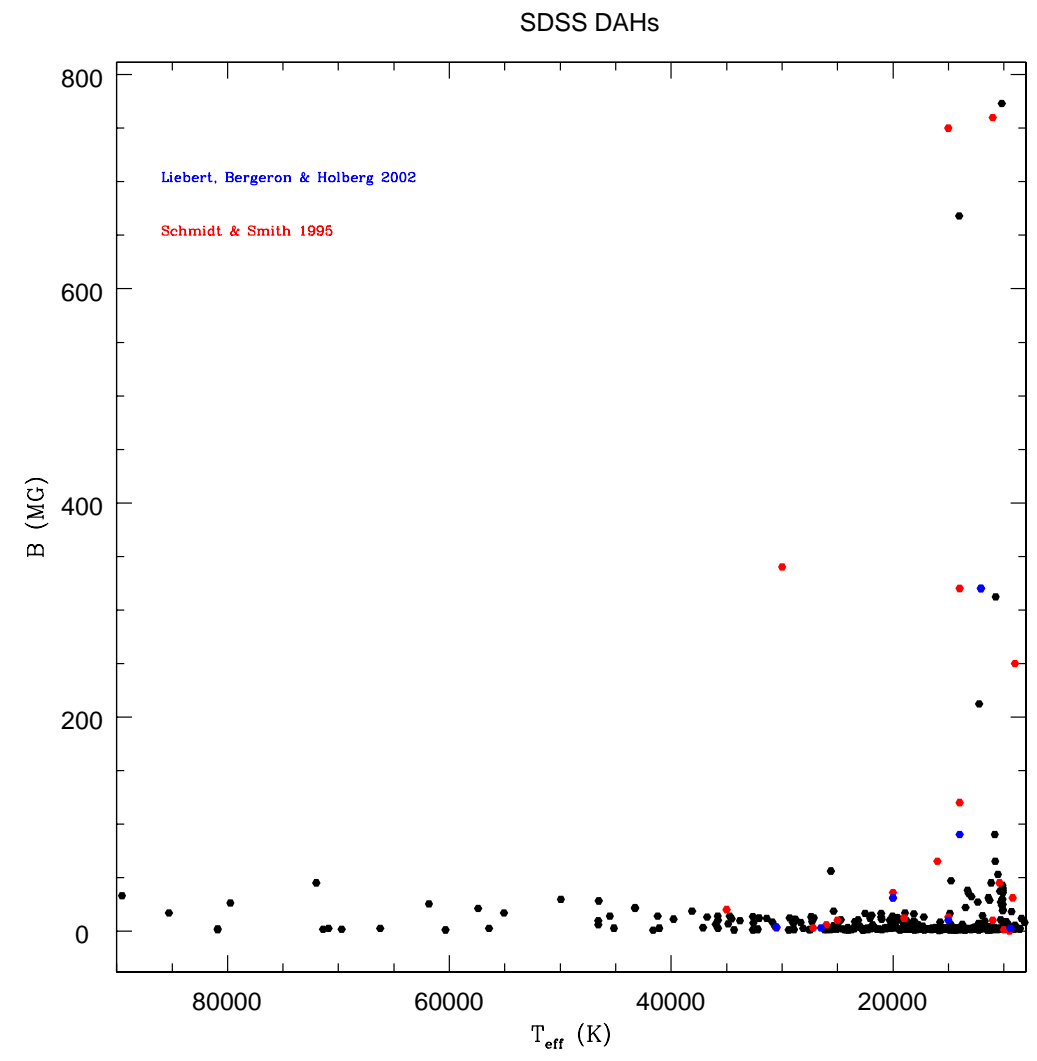

FIGURE 2. Magnetic field versus effective temperature.

For the stars with multiple SDSS spectra, and even when we analyze the subcomponents of the co-added SDSS spectra, we detected small displacements in wavelength of the Zeeman-split components with time, consistent with rotation effects of oblique magnetic field rotators.

Another interesting aspect of our study of SDSS white dwarfs is that for most of the stars that we have fitted non-magnetic models with masses above $1 M_{\odot}$, we have detected Zeeman splittings. It is necessary to verify if the increase in mass could be caused by low magnetic fields, as we detect an increase in the mean field around $13000 \mathrm{~K}$ for DAs, the same position in their cooling tracks where they develop surface convection zones. As we detected Zeeman splitting in our disk integrated spectra for 5\% or more of white dwarfs, which means global organized fields, perhaps even smaller or unorganized fields are the cause for the line broadening.

Weaker magnetic fields in white dwarfs have been studied by Koester et al. [21], who obtained high resolution spectra measurements of the NLTE core of $\mathrm{H} \alpha$ for 28 white 
SDSS $\mathrm{S} / \mathrm{N} \geqq 20 \quad \mathrm{~B} \leqq 3.65$ MG Magnetic White Dwarfs

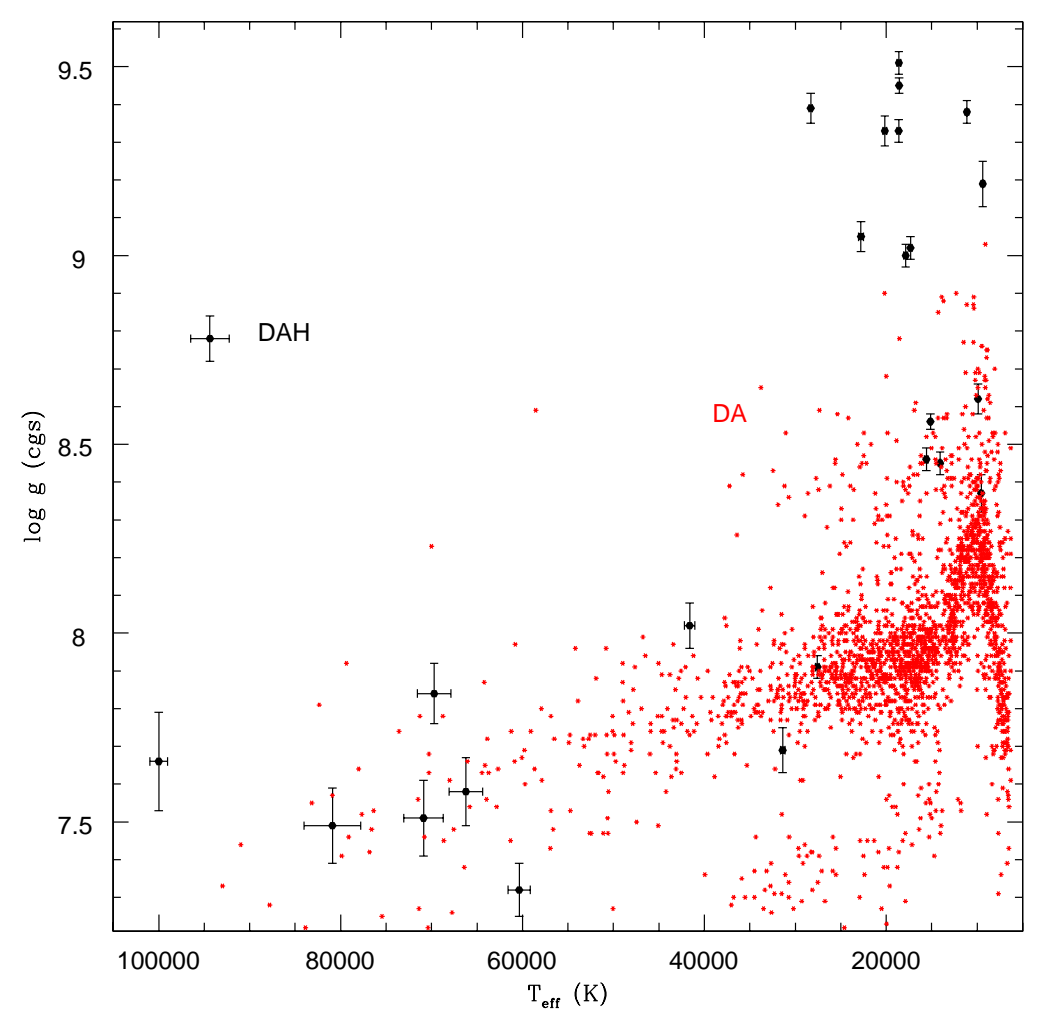

FIGURE 3. Surface gravity estimates assuming no magnetic field versus effective temperature, for stars with $\mathrm{S} / \mathrm{N} \geq 20$, in back for stars with estimated magnetic fields below $3.65 \mathrm{MG}$ and in red with no detected field.

dwarf stars to measure their projected rotational velocities, finding 3 magnetic white dwarfs, no fields above $10-20 \mathrm{kG}$ for the other stars, all hotter than $14000 \mathrm{~K}$. They also found an apparent 30 to $45 \mathrm{~km} / \mathrm{s}$ broadening for 3 pulsating white dwarfs - stars with surface convection. Koester et al. [22] observed about 800 white dwarfs in the SPY survey, finding 10 magnetic, with fields from 3 to $700 \mathrm{kG}$.

For fields larger than $10 \mathrm{kG}$ but smaller than $10 \mathrm{MG}$, i.e., in the Paschen-Back limit, each line will be split into 3 components, with the shifted components separated by around

$$
\Delta \lambda= \pm 4.67 \times 10^{-7} \lambda^{2}
$$

with $\lambda$ in $\AA$ Angstrons and B in MG. For magnetic fields less than $\simeq 1 \mathrm{MG}$, the Zeeman splitting is difficult to observe in low resolution spectra of white dwarfs because the spectral lines are already broadened due to the high density. The linear Zeeman splitting 
corresponds to a broadening of unpolarized spectral lines of the order of $10 \mathrm{~km} / \mathrm{s}$ for fields around $10 \mathrm{kG}$. For higher fields the magnetic energy cannot be included as a perturbation because the cylindrical symmetry of the magnetic field start to disturb the spherical symmetry of the Coulomb force that keeps the hydrogen atom together. The Lorentz force and the Coulomb force are of the same order for B=4670 MG.

The observed Zeeman splitting represents the mean field across the surface of the star. If the field is assumed as dipole, the mean field is related to the polar field by

$$
B=\frac{1}{2} B_{p} \sqrt{1+3 \cos ^{2} \theta}
$$

where $B_{p}$ is the polar field and $\theta$ is the angle between the field and the line of sight.

\section{MEAN MASSES OF NON-MAGNETIC WHITE DWARF STARS}

We excluded the magnetic white dwarfs from our average mass estimate of normal DAs and DBs due to the Zeeman splittings distortions of the line profiles; analysis of the remaining 1505 bright and hot DA white dwarfs, i.e., those with $S / N \geq 20$ and $T_{\text {eff }}=12000 \mathrm{~K}$, results in a mean mass $\langle M\rangle=0.604 \pm 0.003 M_{\odot}$, while that of our 82 bright DBs with $T_{\text {eff }}=16000 \mathrm{~K}$ is $\langle M\rangle=0.646 \pm 0.006 M_{\odot}$.

\section{REFERENCES}

1. B. Külebi et al. 2009, $A \& A, 506,1341$

2. S. Kleinman et al. 2010, in preparation

3. J.-F. Donati \& J. D. Landstreet 2009 ARAA, 47, 333

4. J. Liebert, P. Bergeron \& J. Holberg 2005, ApJS, 156, 47

5. S. O. Kepler et al. 2007, MNRAS, 375, 1315

6. M.-M. Limoges \& P. Bergeron 2010, ApJ, 714, 1037

7. A. Gianninas et al. 2010, ApJ, 720, 581

8. R. E. Falcon, D. E. Winget, M. H. Montgomery, K. A. Williams, 2010, ApJ, 712, 585

9. D. Koester et al. 2009, JPhCS, 172, a2006

10. P.-E. Tremblay et al. 2010, ApJ, 712, 1345

11. J. Liebert et al. 2003, $A J, 124,248$

12. J. Liebert et al. 1988 PASP 100, 1372

13. E. Sion et al. 1988 AJ, 96, 251

14. A. Kawka et al. 2003, NATO Science Series II, 105, 109

15. J. Power et al. 2008, Constrib. Astron. Obs. Skalnate Pleso, 38, 443

16. S. Jordan et al. 2007, A\&A, 462, 1097

17. J. Braithwaite \& H. C. Spruit 2004, Nature, 431, 839

18. C. A. Tout et al. $2004 M N R A S, 355, \mathrm{~L} 13$

19. D. Wickramasinghe \& L. Ferrario 2000, PASP 112, 873

20. D. Wickramasinghe \& L. Ferrario 2005, MNRAS, 356, 1576

21. D. Koester et al. $1998, A \& A, 338,612$

22. D. Koester et al. $2009 A \& A, 505,441$ 
Copyright of AIP Conference Proceedings is the property of American Institute of Physics and its content may not be copied or emailed to multiple sites or posted to a listserv without the copyright holder's express written permission. However, users may print, download, or email articles for individual use. 